Featured graphic

\title{
Visualising Children's Services Data: A composite of the Child Welfare Inequalities Project App
}

\author{
Calum Webb* and Rhiannon Thomas \\ University of Sheffield
}

The most deprived ten per cent of neighbourhoods in England have a ten times higher rate of children in the care system than the least deprived 10 per cent, and a 12 times higher rate of child protection plans (Bywaters, et al. 2018). Levels of deprivation, income inequality, higher education, and ethic density can explain almost 75 per cent of the variation in local authority rates of children in care (Webb, et al. 2021), in part because Black and Mixed Heritage children are 35 per cent to 55 per cent more likely to be in care than White children (Bywaters, et al. 2019) and have four times higher rates of care than the White British population in low deprivation areas (Webb, et al. 2020). Between 2010 and 2015, a rise in expenditure required to sustain a growing and increasingly privatised care system (Office of the Children's Commissioner, 2020) masked enormous reductions in expenditure on preventative services, which fell by 38 per cent on average and by 46 per cent in the most deprived 50 local authorities in England (Webb and Bywaters, 2018). This retrenchment continued throughout the remainder of the decade (Action for Children, 2020).

These statistics alone are dramatic. However, highlighting dramatic inequalities does not necessarily lead to meaningful changes in policy or practice. While all local authorities recognised these inequalities and funding pressures, they often reflected that knowing average trends did not always facilitate challenging them. This was reinforced by political narratives of the effect of 'outstanding leadership and innovation' that sideline structural issues; most local leaders feel they are outstanding but, by definition, only a few can be. To create policy change key actors needed to see the patterns from abstract models reflected in their locality. Similarly, rightly or wrongly, community organisations increasingly need to leverage quantitative data to secure funding or demonstrate a need for services in a particular place.

To meet these needs we developed the Child Welfare Inequalities Project (CWIP) App, from which the featured composite graphic is taken (Webb and Thomas, 2020). The app hosts a repository of 16 merged child welfare and small-area datasets which are then visualised in a variety of ways, with user controls for local authority, variable, time range, and plot types. The app can also run and output results from rudimentary statistical tests and linear models. It is a 'Shiny' app built in the open source statistical programming language R (R Core Team, 2021; Wickham, 2021). At the time of its creation our only familiarity with programming was applied statistical analysis; the app reflects how valuable frameworks like Shiny and resources from the R community are for enabling researchers to make something that might previously have taken months of familiarisation with unfamiliar scripting languages. The app itself is open-source[1] and medium, provided the original author and source are credited. 
can be accessed in-browser.[2] It is hosted on Rstudio's shinyapps.io platform and has been in 'active use' for over 1,000 hours since release.

The composite graphic highlights three features. Row A shows the 'dashboard', which allows users to visualise trends over time (left) with accompanying plots (right) which, in this case, show the association between deprivation and rising rates of children in care. Row B illustrates how changes in children's services expenditure can be visualised through 'waffle charts'. Lastly, Row C shows the app's ability to create detailed bivariate maps - intersections between small-area variables that can show, for instance, that the majority of areas with high rates of child poverty are often the same areas where a relatively large proportion of the population identify as Black/Black British, highlighting issues of structural racism as expressed through place. Thousands of possible data visualisations can be created with no programming experience, allowing users to both better contextualise our research and explore their own questions further.

On a perfunctory level, this use of interactive visualisation shortens the distance between research and the communities for whom we believe it matters. More fundamentally, it allows people to ask their own questions of the data. Static visualisations are an important but limited medium; they are a monologue between researchers and 'audiences', not a dialogue. The kinds of questions they address are often those of people already in positions of considerable privilege - those with the capital to commission or pursue them. If we truly want to 'open' quantitative research, it is important to ask how everyone can fully participate. The CWIP App was a modest attempt to build additional bridges between the national and local policy implications of our research, and illustrates the potential of tools like Shiny that fit easily into the increasingly common $\mathrm{R}$ workspace of quantitative social scientists. 
p. 102. Visualising Children's Services Data: A composite of the Child Welfare Inequalities Project App

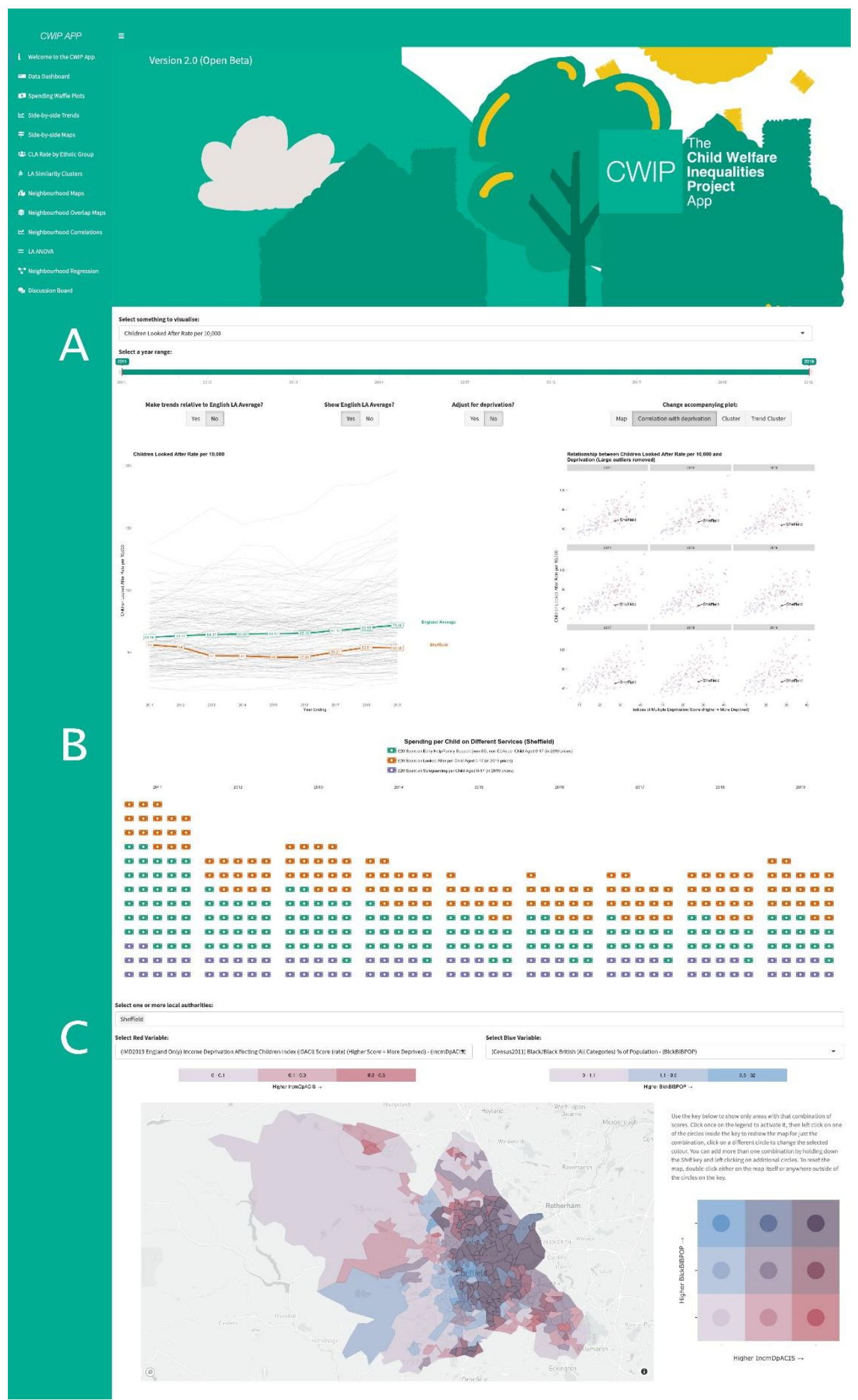




\section{Notes}

[1] https://github.com/cjrwebb/cwip-app

[2] https://www.cwip-app.co.uk

*Correspondence address: Calum Webb, Department of Sociological Studies, Elmfield, Northumberland Road, Sheffield, S10 2TU. Email: c.j.webb@sheffield.ac.uk

\section{References}

Action for Children (2020) Children and young people's services: funding and spending 2010/11 to 2018/19. London: Action for Children. Available at: https://media.actionforchildren.org.uk/documents/Joint report childrens services funding 2018-19 May 2020 Final.pdf

[Accessed: 27/04/2021]

Bywaters, P., Scourfield, J., Webb, C., Morris, K.M., Featherstone, B., Brady, G., Jones, C. and Sparks, T. (2019) Paradoxical evidence on ethnic inequities in child welfare: towards a research agenda. Children and Youth Services Review, 96, 145-154.

Bywaters, P., Brady, G., Bunting, L., Daniel, B., Featherstone, B., Jones, C., Morris, K., Scourfield, J., Sparks, T. and Webb, C. (2018) Inequalities in English child protection practice under austerity: A universal challenge? Child \& Family Social Work, 23, 1, 53-61.

Office of the Children's Commissioner (2020) Private provision in children's social care. London: Children's Commissioner for England. Available at: https://ccoweb.azureedge.net/wp-content/uploads/2020/11/cco-private-provision-inchildrens-social-care.pdf [Accessed: 27/04/2021]

R Core Team (2021) R: A Language and Environment for Statistical Computing. Vienna: R Foundation for Statistical Computing.

Webb, C.J. and Bywaters, P. (2018) Austerity, rationing and inequity: trends in children's and young peoples' services expenditure in England between 2010 and 2015. Local Government Studies, 44, 3, 391-415.

Webb, C., Bywaters, P., Scourfield, J., Davidson, G. and Bunting, L. (2020) Cuts both ways: Ethnicity, poverty, and the social gradient in child welfare interventions. Children and Youth Services Review, 117, 105299.

Webb, C. and Thomas, R. (2020) The Child Welfare Inequalities Project App. Available at: https://www.cwip-app.co.uk [Accessed: 27/04/2021]

Webb, C.J.R., Bywaters, P., Elliott, M. and Scourfield, J. (2021) Income inequality and child welfare interventions in England and Wales. Journal of Epidemiology and Community Health, 75, 3, 251-257.

Wickham, H. (2021) Mastering Shiny: Build Interactive Apps, Reports, and Dashboards Powered by R. Farnham: O'Reilly. 